\title{
Measurement Uncertainty Assessment in Remote Object Geolocation
}

\author{
Lovro Kuščer* - Janez Diaci \\ University of Ljubljana, Faculty of Mechanical Engineering, Slovenia
}

Remote object geolocation is a process of determining the geographic location of distant objects, which is often required in geology, military applications, surveying and elsewhere. Unlike in the case of widely used satellite localization and navigation solutions, there are very few studies of the measurement uncertainties and their propagation in the case of geolocating distant, non-cooperative objects. To investigate this specific segment of navigation we developed an experimental system built entirely of COTS (Commercial Of-The-Shelf) components. The aim of this work is to study the possibility for assessing the measurement characteristics of such integrated systems, based on the specified uncertainties of the individual measuring devices.

Keywords: remote object geolocation, measurement uncertainty, Monte Carlo simulation

\section{O INTRODUCTION}

Global navigation satellite systems (GNSS) provide the means for fast determination of the geographic location, speed and time. Combined with the modern satellite receivers they enable high positioning precision and reliability in various environments. However, it is sometimes not sufficient to precisely establish only the position of the receiver. In some circumstances it is necessary to obtain the position of a distant, inaccessible or hazardous object. That is where the demand for the remote object geolocation arises. The capability of the remote geolocation is often required in geology [1], military applications [2] and [3], surveying [4], agriculture, forestry [5] and elsewhere.

Many research works investigated different techniques for acquiring accurate remote object (or target) positions from ground or unmanned air vehicles (UAV) [6] and [7]. In order to obtain the target geolocation the UAV systems commonly employ a gimbal camera, a geo-referenced terrain database and a navigation system. Some advanced aerial systems employ SAR (Synthetic Aperture Radar) which allows high resolution imaging at long stand-off ranges [3]. In the case of target geolocation from the ground, laser rangefinders combined with satellite and inertial navigation systems [8] to [11] are often utilized.

The main challenge in remote object localization is to precisely measure the position and attitude of the measuring system. While the estimation of position (using satellite navigation systems) is generally straightforward, the attitude determination with sufficient accuracy can be more difficult. The associated measurement uncertainties of the position [12] and especially the attitude measurements [13] contribute significantly to the evaluated remote object's position uncertainty.

To study the options for remote object geolocation we designed and built an experimental measuring system using only COTS (Commercial Of-TheShelf) components. In this article we investigate to what extent it is possible to predict the measurement uncertainty of the integrated measuring system based on the manufacturer specifications for each employed COTS device. We also present the experimental work aimed at determining the actual measuring characteristics and examine the opportunities for enhancing the overall performance at measuring distances up to $20 \mathrm{~km}$.

\section{GEOLOCATING REMOTE OBJECTS}

The following section presents a brief overview of the method used for determining the geographic location of a remote object in the World Geodetic System 1984 (WGS 84). The WGS 84 coordinate system [14] was selected since it is currently used as the reference coordinate system for the Global Positioning System (GPS).

The first step is the determination of the geographic location of the measuring system (origin) which is given by its latitude, longitude and altitude $\left(\varphi_{0}, \lambda_{0}, h_{\mathrm{o}}\right)$ in WGS 84 coordinate system. Next, the relative position of the remote object with respect to the origin is obtained. The relative position is given by the distance $\left(l_{o r}\right)$, azimuth $\left(\alpha_{o r}\right)$ and elevation $\left(\beta_{o r}\right)$ in a spherical coordinate system (Fig. 1).

Since the input coordinates (origin, relative position of the remote object) are given in two different coordinate systems, we transform them to a common coordinate system in order to calculate the geographic location of the remote object. For this 
purpose the WGS 84 Cartesian coordinate system is used. This is a right-handed Cartesian coordinate system with the origin at the Earth's centre of mass, the $Z$-axis in the direction of the IERS (International Earth Rotation and Reference Systems Service) reference pole, the $X$-axis as the intersection of the IERS reference meridian and the plane through the origin normal to the $Z$-axis, and the $Y$-axis completing the right-handed orthogonal coordinate system (Fig. 2a). The transformation of the WGS 84 coordinates from latitude, longitude and altitude to the Cartesian coordinates is given by Eq. (1) [15] where $x_{o}, y_{o}$ and $z_{\mathrm{o}}$ are the Cartesian coordinates of the origin, $N$ is the radius of curvature in the prime vertical, $e$ is the first eccentricity, and $a$ and $b$ are the semimajor and semiminor axis of the WGS 84 reference ellipsoid, respectively.

$$
\begin{gathered}
\left(\begin{array}{l}
x_{o} \\
y_{o} \\
z_{o}
\end{array}\right)=\left(\begin{array}{c}
\left(N+h_{o}\right) \cos \varphi_{o} \cos \lambda_{o} \\
\left(N+h_{o}\right) \cos \varphi_{o} \sin \lambda_{o} \\
\left(N\left(1-e^{2}\right)+h_{o}\right) \sin \varphi_{o}
\end{array}\right), \\
N=\frac{a}{\sqrt{1-e^{2} \sin ^{2} \varphi_{o}}}, \quad e=\frac{\sqrt{a^{2}-b^{2}}}{a} .
\end{gathered}
$$



Fig. 1. Determination of the geographic location of a remote object

Next, the Eq. (2) is used to transform the relative position of the remote object (given in spherical coordinates) to the Cartesian coordinates $\left(x_{l o c}, y_{l o c}\right.$, $\left.z_{l o c}\right)$ in a local coordinate system (Fig. 2b).

$$
\left(\begin{array}{c}
x_{l o c} \\
y_{l o c} \\
z_{l o c}
\end{array}\right)=\left(\begin{array}{c}
-l_{o r} \cos \beta_{o r} \cos \alpha_{o r} \\
l_{o r} \cos \beta_{o r} \sin \alpha_{o r} \\
l_{o r} \sin \beta_{o r}
\end{array}\right) .
$$

The coordinates in the local Cartesian coordinate system are then converted to the offset in the WGS 84
Cartesian coordinate system. By adding this offset to the Cartesian coordinates of the origin, the coordinates of the remote object are obtained (Eq. (3)).

$$
\left(\begin{array}{l}
x_{r} \\
y_{r} \\
z_{r}
\end{array}\right)=\left(\begin{array}{l}
x_{o} \\
y_{o} \\
z_{o}
\end{array}\right)+
$$

$+\left(\begin{array}{c}x_{l o c} \sin \varphi_{o} \cos \lambda_{o}-y_{l o c} \sin \lambda_{o}+z_{l o c} \cos \varphi_{o} \cos \lambda_{o} \\ x_{l o c} \sin \varphi_{o} \sin \lambda_{o}+y_{l o c} \cos \lambda_{o}+z_{l o c} \cos \varphi_{o} \sin \lambda_{o} \\ z_{l o c} \sin \varphi_{o}-x_{l o c} \cos \varphi_{o}\end{array}\right)$.

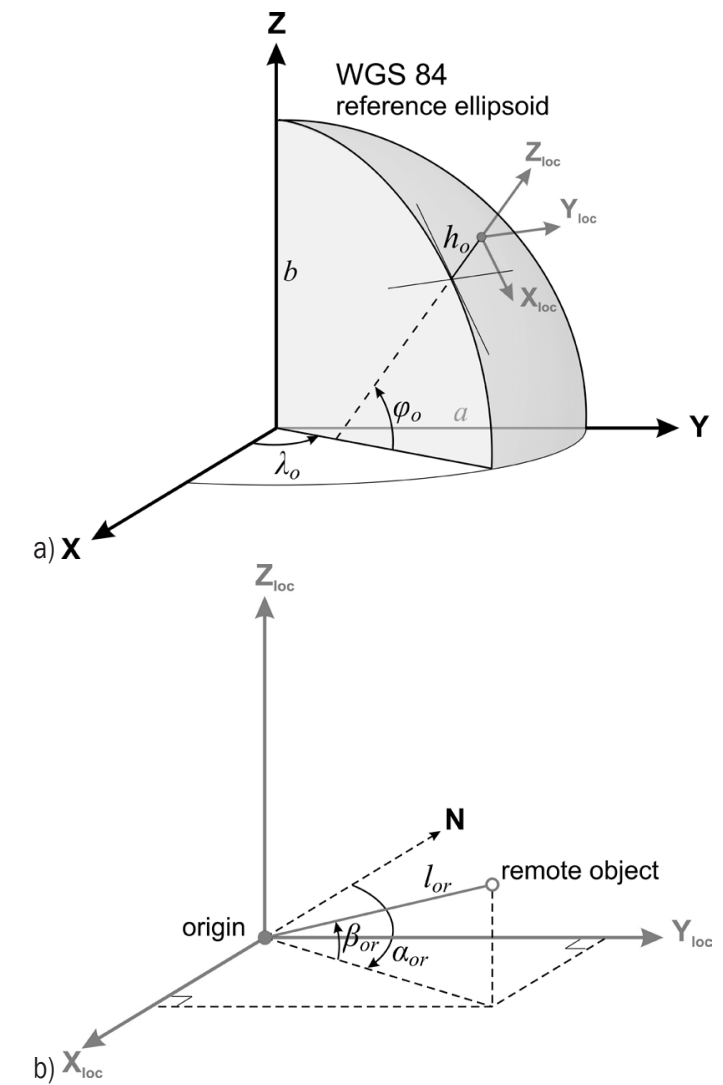

Fig. 2. Coordinate systems in remote object geolocation; a) WGS 84 coordinate system, b) local coordinate system

Finally, the Cartesian coordinates of the remote object $\left(x_{r}, y_{r}, z_{r}\right)$ are transformed back to the latitude, longitude and altitude $\left(\varphi_{r}, \lambda_{r}, h_{r}\right)$ in the WGS 84 coordinate system. This task is solved numerically by utilizing the Hirvonen and Moritz iterative method [16]. The described procedure yields the remote object's coordinates in the WGS 84 coordinate system.

\section{EXPERIMENTAL SYSTEM}

The experimental system is designed for use on land vehicles and consists of an inertially stabilized pan-tilt 
unit, a laser rangefinder, a GNSS receiver/compass, an electronic inclinometer and a CCTV video camera (Fig. 3). The system is controlled using a portable computer with custom software.

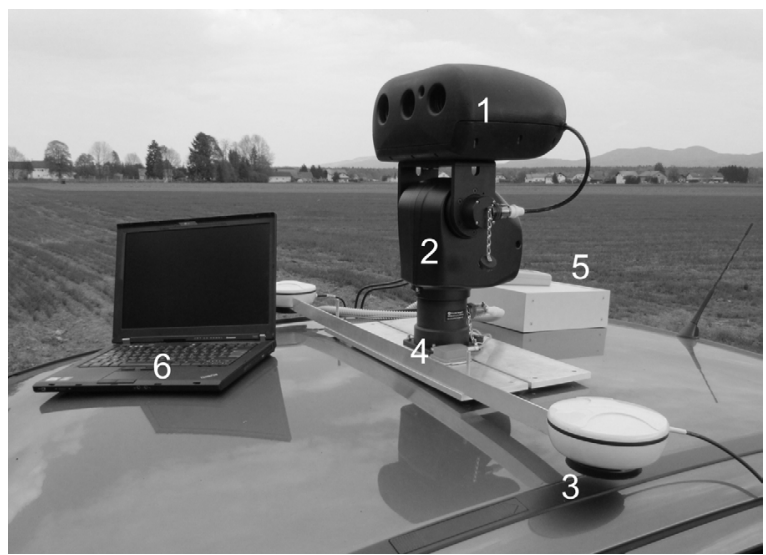

Fig. 3. Experimental system: (1) the laser rangefinder and video camera unit, (2) the pan-tilt unit, (3) the GNSS antenna, (4) the electronic inclinometer, (5) the communication and power interface, (6) the portable computer/controller

The laser rangefinder with a measuring range of $50 \mathrm{~m}$ to $20 \mathrm{~km}$ and the video camera are mounted on the pan-tilt unit, whereas the GNSS receiver/compass antennas and electronic inclinometer are attached to the platform base. Due to this setup, the distance $\left(l_{o r}\right)$ and origin geographic location $\left(\varphi_{o}, \lambda_{o}, h_{o}\right)$ are obtained directly from the rangefinder and the GNSS receiver readings, while the azimuth $\left(\alpha_{o r}\right)$ and elevation $\left(\beta_{o r}\right)$ are calculated employing the pan and tilt angles of the pan-tilt unit and the platform base orientation. The later is measured with the GNSS compass (yaw) and the electronic inclinometer (pitch and roll).

To carry out the calculation of the azimuth and elevation from the measured quantities, we define a right-handed Cartesian coordinate system with the $X$-axis in the direction of the rangefinder laser beam and the $Y$-axis parallel with the tilt axis of the pan-tilt unit. In this coordinate system, we define a unit vector $v$ that points in the direction of the positive $X$-axis. Next, we perform five consecutive coordinate system rotations to account for the tilt $(\theta)$, pan $(\delta)$, roll $(\gamma)$, pitch $(\beta)$, and yaw $(\alpha)$ by utilizing Eq. (4):

$$
v^{\prime}=R_{z}(\alpha) R_{y}(\beta) R_{x}(\gamma) R_{z}(\delta) R_{y}(\theta) v,
$$

where:

$$
R_{x}(\alpha)=\left[\begin{array}{ccc}
1 & 0 & 0 \\
0 & \cos (\alpha) & \sin (\alpha) \\
0 & -\sin (\alpha) & \cos (\alpha)
\end{array}\right],
$$

$$
\begin{aligned}
& R_{y}(\alpha)=\left[\begin{array}{ccc}
\cos (\alpha) & 0 & -\sin (\alpha) \\
0 & 1 & 0 \\
\sin (\alpha) & 0 & \cos (\alpha)
\end{array}\right], \\
& R_{z}(\alpha)=\left[\begin{array}{ccc}
\cos (\alpha) & \sin (\alpha) & 0 \\
-\sin (\alpha) & \cos (\alpha) & 0 \\
0 & 0 & 1
\end{array}\right] .
\end{aligned}
$$

In Eq. (4), the $R_{x}, R_{y}$ and $R_{z}$ are the rotation matrices for the coordinate system rotation about $x, y$ and $z$, respectively and $v^{\prime}$ is the representation of the unit vector $v$ in the transformed coordinate system. From the vector $v$, the azimuth and elevation are obtained using Eq. (6):

$$
\begin{gathered}
\alpha_{o r}=\arctan \left(\frac{v_{y}{ }^{\prime}}{v_{x}{ }^{\prime}}\right), \\
\beta_{o r}=\arcsin \left(v_{z}{ }^{\prime}\right),
\end{gathered}
$$

where the arctan is the four quadrant arctangent and $v_{x}{ }^{\prime}, v_{y}{ }^{\prime}$ and $v_{z}{ }^{\prime}$ are the coordinates of the vector $v^{\prime}$.

\section{MEASUREMENT CHARACTERISTIC}

The measurement performance of the system depends on the measurement uncertainties of the employed devices. The manufacturer specification of the measuring equipment in the experimental system is presented in Table 1.

Table 1. Manufacturer specification for measuring equipment

\begin{tabular}{ccccc}
\hline $\begin{array}{c}\text { Measuring } \\
\text { equipment }\end{array}$ & $\begin{array}{c}\text { Laser } \\
\text { rangefinder }\end{array}$ & $\begin{array}{c}\text { GNSS } \\
\text { positioning } \\
\text { module }\end{array}$ & $\begin{array}{c}\text { GNSS } \\
\text { compass }\end{array}$ & $\begin{array}{c}\text { Electronic } \\
\text { inclinometer } \\
\text { (pitch and roll) }\end{array}$ \\
\hline $\begin{array}{c}\text { Containment } \\
\text { limits }\end{array}$ & $\pm 3 \mathrm{~m}$ & $\begin{array}{c}0.60 \mathrm{~m} \\
\text { (DGPS) }\end{array}$ & $\pm 0.15^{\circ}$ & $\pm 0.5^{\circ}$ \\
\hline $\begin{array}{c}\text { Containment } \\
\text { probability }\end{array}$ & $95 \%$ & $95 \%$ & $95 \%$ \\
\hline Latitude: \\
$10-5$ ' \\
Longitude: \\
$10-5$ ' \\
Resolution
\end{tabular}

The uncertainty of each individual measurement contributes to the derived position uncertainty of the remote object in a specific manner. There are several methods for evaluating the combined effect of the individual measurement uncertainties on the result. The law of propagation of variances is most commonly used when the result can be calculated with a closed form expression. In the case of nonlinear 
relations, the system equations are first linearized using the Taylor series [17]. However, in this case, a numerical solution (Hirvonen and Moritz iterative method) is employed to determine the position of the remote object. Hence, we use the Monte Carlo simulation [18] to [21] to assess the propagation of the measurement uncertainties.

The term Monte Carlo denotes a set of stochastic computational techniques that are widely used for computer simulations in various fields of science and engineering. In the case of probabilistic uncertainty analysis, a probability density function is assigned to each measuring device. Then, repeated numerical calculations of the remote object's position using randomly selected values from the assigned distributions are performed. This basically simulates the execution of multiple measurements in the presence of measurement uncertainties. The calculated positions of the remote object form a distribution which represents the combined effect of each measuring device and its associated uncertainty. The statistical analysis of the obtained distribution yields an estimation of the measurement uncertainty in the remote object's position.

\section{SIMULATION}

To perform the simulation the uncertainty estimates of the individual measuring devices need to be obtained. According to [22] this can be achieved by two different approaches. The first one (Type A) is based on retrieving relevant statistical information form repeated measurements, while the second (Type B) relies on manufacturer specification, past experience, or other sources. Since we are investigating the possibility of predicting, and not measuring the uncertainty of an integrated system, we utilize Type B estimate.

The manufacturers often specify the uncertainty of their measuring equipment by the error containment limits and containment probability, while the underlying distribution is usually not provided. Commonly, the normal distribution is assumed applicable for the combined error of the measuring equipment with a central tendency and symmetrical containment limits. Since there is no indication that would encourage the use of a different probability distribution, we assume the normal distribution of the combined error for all measuring devices.

When the error is normally distributed, the uncertainty $(u)$ is obtained from Eq. (7) [23] where $\pm L$ represents the containment limits, $p$ the containment probability, and $\Phi^{-1}()$ the inverse normal distribution function.

$$
u=\frac{L}{\Phi^{-1}\left(\frac{1+p}{2}\right)} .
$$

By applying Eq. (7) to the manufacturer specifications for error containment limits and containment probability (Table 1), the standard uncertainties of $1.5 \mathrm{~m}$ for distance measurements, 0.3 $\mathrm{m}$ for position of the origin, $0.15^{\circ}$ for azimuth, and $0.26^{\circ}$ for elevation are obtained.

In order to illustrate the key characteristics of the results of Monte Carlo simulation Table 2 presents measurement uncertainties at four measuring distances $(100 \mathrm{~m}, 1,10$ and $20 \mathrm{~km})$ and $0.0^{\circ}$ elevation angle. The uncertainties have been determined using 10,000 simulation runs for each distance. To facilitate the discussion the combined 2D uncertainty is presented along with its constituents in two separate directions denoting as longitudinal and lateral the direction of the rangefinder laser beam and its orthogonal, respectively. According to [23] the presented uncertainties are equivalent to the standard deviations of the error distributions and are evaluated by analyzing the simulation points. From the reported results it is evident that the increase in the measuring distance significantly influences the uncertainty in the lateral direction, while the one in longitudinal direction remains virtually constant for the simulated distances.

The stated combined uncertainty enables the evaluation of confidence limits and confidence levels at certain measurement distance. For example, by multiplying the combined uncertainty of $3.1 \mathrm{~m}$ (object at $1 \mathrm{~km}$ distance) with the coverage factor of 2 , we obtain the expanded uncertainty of $\pm 6.2 \mathrm{~m}$ with a confidence level of approximately 95\% (assuming the errors are normally distributed). In other words, $95 \%$ of all measurements performed at $1 \mathrm{~km}$ stand-off range are expected to fall within a circle with a $6.2 \mathrm{~m}$ radius around the true position of the distant object.

The expected impact of the elevation uncertainty on the $2 \mathrm{D}$ combined uncertainty is minimal at the $0.0^{\circ}$ elevation angle. When increasing the elevation angle, its uncertainty increasingly affects the resulting uncertainty in longitudinal direction. This effect is demonstrated in Fig. 4a, which shows the dependence of the uncertainty in longitudinal direction on the measuring distance and elevation angle.

Despite the evident influence of the elevation angle value on its uncertainty propagation, this 

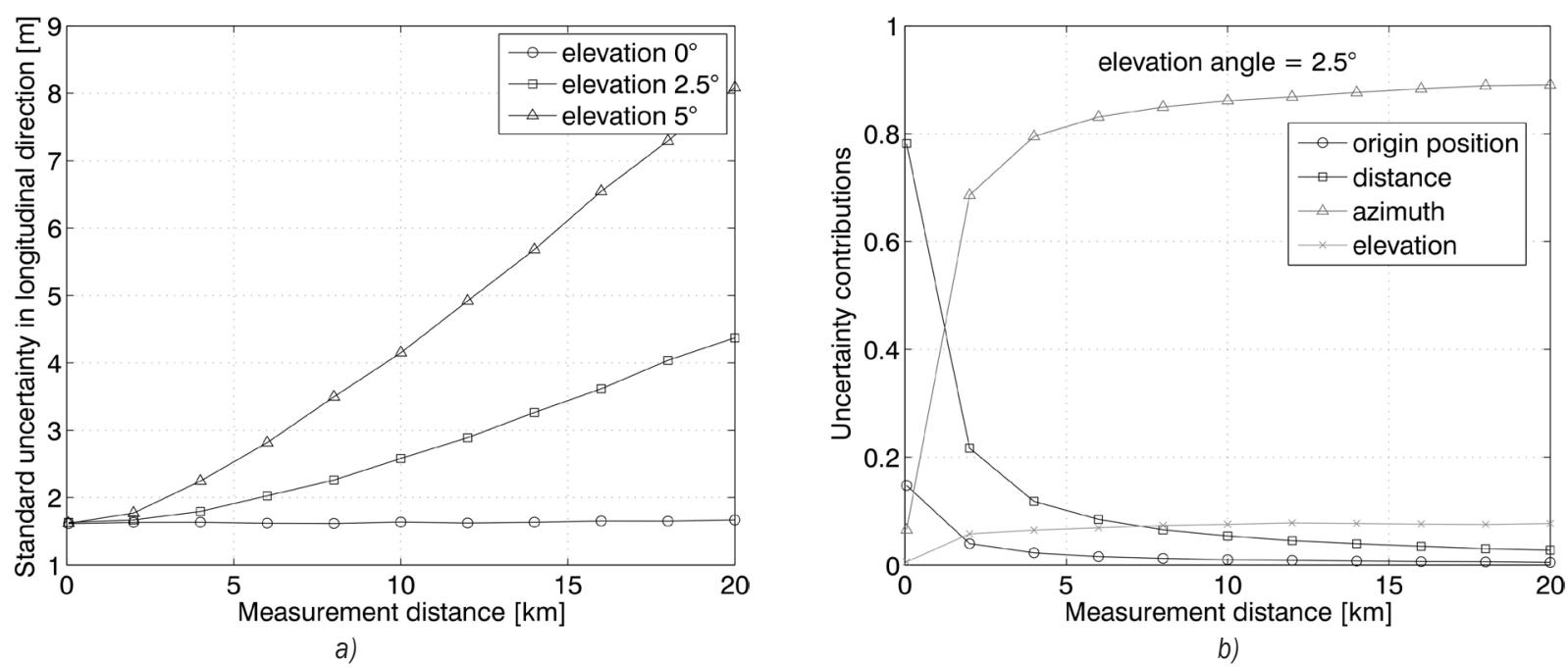

Fig. 4. a) Dependence of the standard uncertainty in longitudinal direction on the measurement distance at different elevation angles, and b) dependence of the relative uncertainty contributions of the simulated quantities on the measurement distance

only contributes a rather small part to the combined uncertainty of the remote object's position. This is clearly seen in Fig. 4b, where the relative contributions of the uncertainties in the simulated quantities to the overall measurement uncertainty are presented. The contributions are calculated for the elevation angle of $2.5^{\circ}$ and are normalized to the sum of the resulting uncertainties of the individual quantities. It has been noticed that at distances below $1 \mathrm{~km}$ the most significant uncertainty source is attributed to distance measurement, while at greater distances the azimuth uncertainty prevails. Furthermore, the amount of the elevation uncertainty contribution, which highly depends on the elevation angle value (Fig. 4a), becomes the second most important uncertainty source at distances above $10 \mathrm{~km}$.

Table 2. Results of the simulation-based uncertainty analysis

\begin{tabular}{llcccc}
\hline & Distance & $100 \mathrm{~m}$ & $1 \mathrm{~km}$ & $10 \mathrm{~km}$ & $20 \mathrm{~km}$ \\
\cline { 2 - 6 } & Elevation & $0.0^{\circ}$ & $0.0^{\circ}$ & $0.0^{\circ}$ & $0.0^{\circ}$ \\
\hline \multirow{2}{*}{$\begin{array}{l}\text { Standard } \\
\text { uncertainty [m] }\end{array}$} & Longitudinal & 1.6 & 1.6 & 1.6 & 1.7 \\
\cline { 2 - 6 } & Lateral & 0.4 & 2.6 & 26.1 & 52.2 \\
\hline Combined uncertainty [m] & 1.7 & 3.1 & 26.2 & 52.2 \\
\hline
\end{tabular}

\section{EXPERIMENTAL RESULTS AND} COMPARISON WITH SIMULATION

A series of field tests were performed in order to verify the simulation results. An open field with an unobstructed view of the sky was used as the test polygon (Fig. 5) with four distant objects located at different distances from the measuring system. The closest two objects were set up for the needs of the experiments $(1 \times 1 \mathrm{~m}$ targets $)$, whereas two existing objects were used for long distance measurements. The positions of the closest two objects were determined using a high performance GNSS receiver also exploiting the corrections from the EGNOS (European Geostationary Navigation Overlay Service) satellite based augmentation system (SBAS).

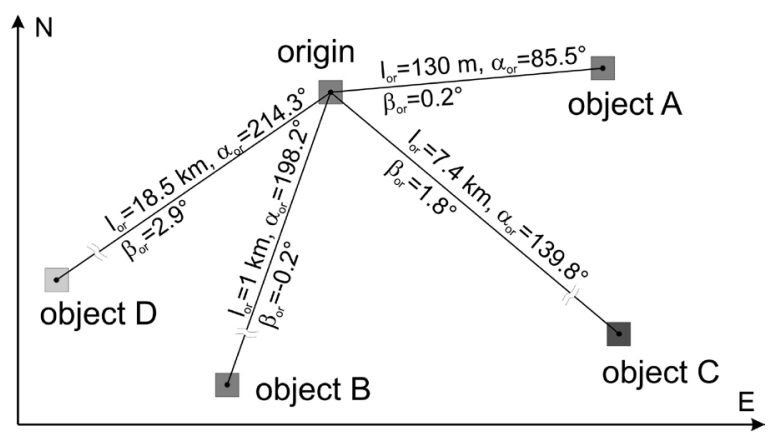

Fig. 5. Layout of the test polygon

The measurements were performed over a period of 30 minutes with an unobstructed view of the sky. According to the manufacturer specification of the GNSS receiver and the available data on EGNOS system, it is reasonable to assume that the standard uncertainty of the measured 2D positions is about $1 \mathrm{~m}$. The positions of the existing distant objects were obtained from the digital orthophoto images (DOF050) [24] with the specified standard uncertainty of $\pm 1 \mathrm{~m}$. Once the existing objects were identified in the digital image, their WGS 84 coordinates were determined employing the geographic information system. 


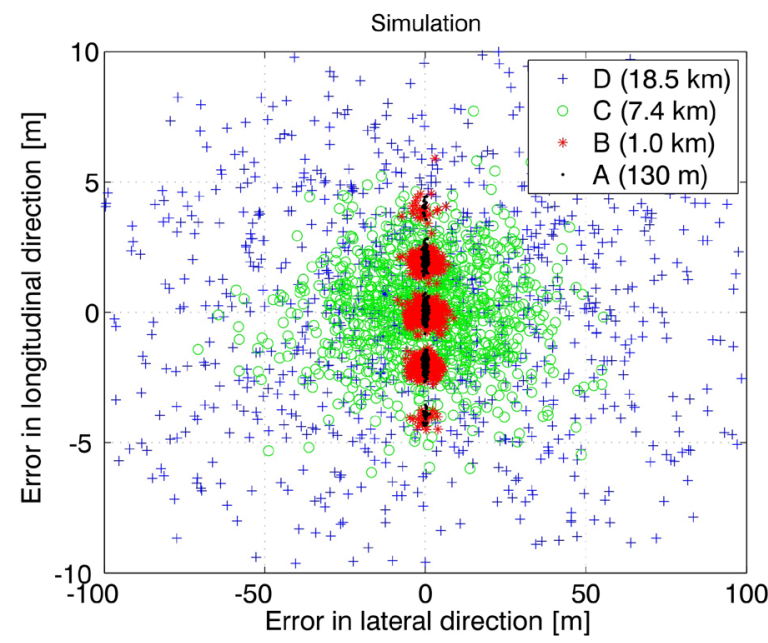

a)

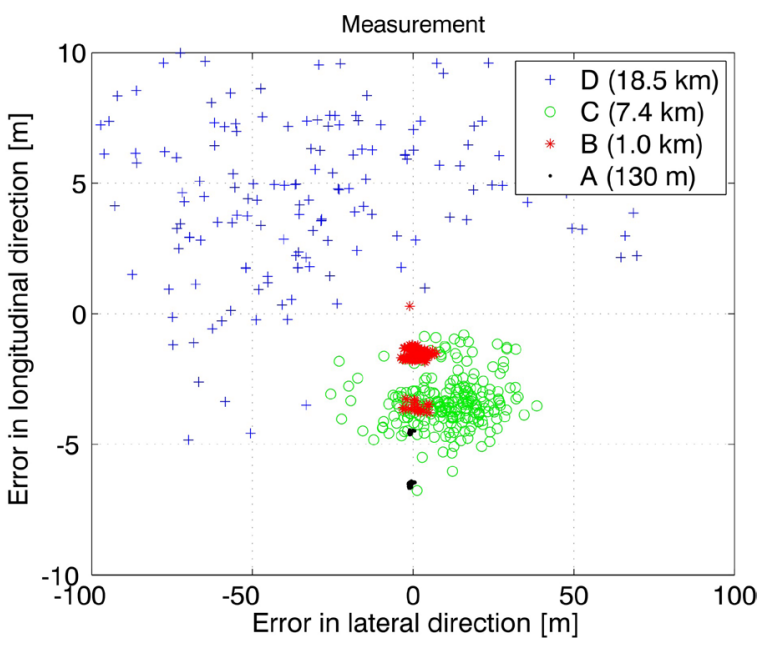

b)

Fig. 6. Distributions of errors obtained by; a) Monte Carlo simulation and b) field tests

The distant objects were positioned at distances of $130 \mathrm{~m}$ (object A), $1.0 \mathrm{~km}$ (object B), $7.4 \mathrm{~km}$ (object C) and $18.5 \mathrm{~km}$ (object D) from the measuring system. The corresponding azimuths of the objects A, B, C, and $\mathrm{D}$ (observed from the origin) were 85.5, 198.2, 139.8 , and $214.3^{\circ}$, respectively.

Since the aim is to investigate the uncertainty in instantaneous geolocation of distant objects, all measurements were performed by employing only the current readings from the sensors without averaging over time. The period of measurements (limited by the repetition rate of the laser rangefinder) was about 6 s. Approximately 200 measurements were performed for each distant object over a period of 20 minutes. Moreover, we performed a Monte Carlo simulation with the distances, azimuths and elevations of the four objects in the test polygon. The simulation results along with the field test results are displayed in Fig. 6. In addition, the simulation output was compared with the results obtained by the use of the law of propagation of variances.

We notice that the simulation and measurement points for objects A and B in Fig. 6 are not distributed evenly but are rather grouped in several clusters. This is caused by the $2 \mathrm{~m}$ resolution of the range measurements which is also considered in the simulation. However, this effect is not observed for the objects $\mathrm{C}$ and $\mathrm{D}$ because of larger distances and the elevation angles of 1.8 and $2.9^{\circ}$. Due to the increased distance and elevation angle the measurement uncertainty of the electronic inclinometer becomes more pronounced (Fig. 4a) and overrides the effect of low range measurement resolution. When comparing the simulation and measurement results in Fig.
6 , it is evident that a considerable measurement bias is present in the longitudinal direction. The observed discrepancies originate from the distance measurements that tend to be smaller than the actual distances for objects $\mathrm{A}, \mathrm{B}$, and $\mathrm{C}$ and larger for object D. However, these discrepancies appear to be systematic and can therefore be corrected for by the calibration over the entire measuring range in order to obtain the distance measurement characteristic that is consistent with the manufacturer specification.

Another distinct difference between the measurements and simulation outputs is the scatter in longitudinal direction for objects A and $\mathrm{B}$, which is considerably larger in the simulation. This is manifested through the occurrence of more separate clusters of simulation points and in larger sizes of individual clusters. Such differences imply that the actual standard deviations of range measurements and origin position measurements are smaller than the assessment based on the manufacturer specifications. Notwithstanding the fact that the discussed discrepancies in longitudinal direction are obvious, they only contribute a small part to the combined uncertainty at larger measuring distances. In these cases, the uncertainty in azimuth measurements gives rise to large errors in lateral direction as can be seen in Fig. 6.

The uncertainty analysis was also performed employing the law of propagation of variances. The results of this approach are displayed in Fig. 7 as error ellipses with 95\% confidence level. Usually, we assume that the underlying error distribution represented by the error ellipse is normal. In the case of objects C and D (Fig. 7b) this assumption seems 


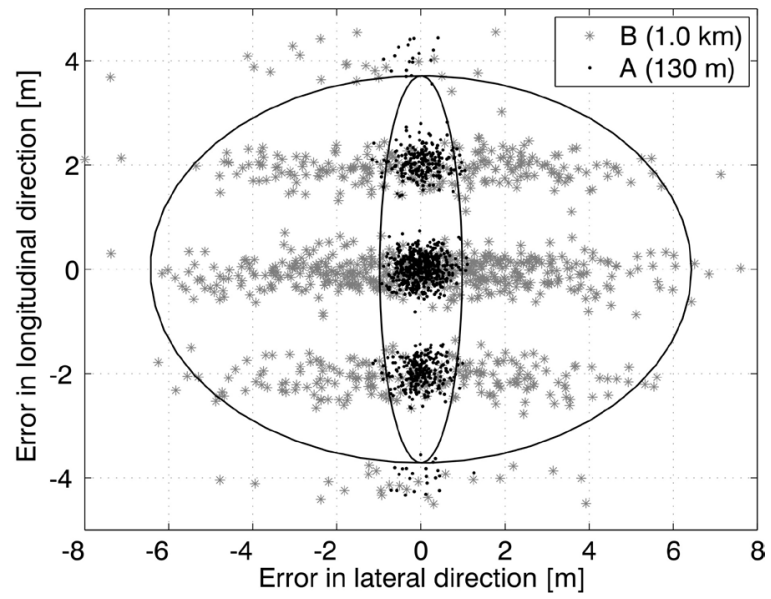

a)

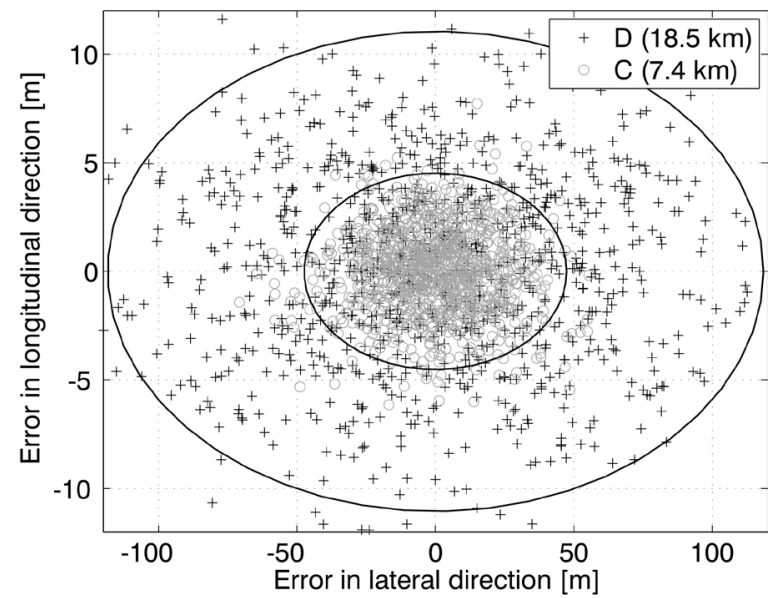

b)

Fig. 7. Distributions of errors obtained by Monte Carlo simulation and error ellipses ( $95 \%$ confidence level) obtained by the law of propagation of variances; a) objects $A$ and $B, b)$ objects $C$ and $D$

justified. However, the errors in Fig. 7a are not normally distributed and might therefore require a different representation.

To describe and examine the distributions obtained by means of simulation and measurements, it is convenient to use standard deviation as a measure of dispersion and root mean square error (RMSE) as a measure of the overall performance. For the simulation, the standard deviations are equivalent to the standard uncertainties reported in Table 2. Furthermore, since no bias is incorporated in the simulation, the RMSE is equivalent to the combined uncertainty, which is in fact the standard deviation of the combined error distribution.

A comparison of the simulation and the field test results (Table 3) indicates that the standard deviations of the measurements in longitudinal direction are significantly smaller than the simulated one for all objects. This implies that the random component of the range measurement error is actually smaller than expected. On the other hand, the systematic component associated with range measurements has a considerable influence on the overall position accuracy, especially at small stand-off ranges (object A).

When comparing the standard deviations in lateral direction it can be noticed that the measurements and the simulations yield similar results only for smaller distances. At longer distances the azimuth measurement uncertainty, which is affected by many factors, including the number of GNSS satellites in view, satellite geometry and ionospheric activity, causes less predictable results. Nevertheless, the difference between the simulated and measured RMSE is less than $5 \mathrm{~m}$ for objects $\mathrm{A}, \mathrm{B}$ and $\mathrm{C}$.

Although the errors in longitudinal direction may have very little impact at long distances, they still limit the system's performance at closer ranges. This is demonstrated in Fig. 8, where we notice that the smallest mean error is not observed at the closest object A but at object B. This is attributed primarily to the aforementioned bias in the distance measurements. However, in general, the measured positions of the more distant objects exhibit larger variations and mean errors compared to the closer ones. This is especially evident when comparing measurements of objects $\mathrm{C}$ and D. Such results are expected considering the dependence of the azimuth and elevation uncertainty propagation on the measuring distance.

The presented results suggest that at smaller stand-off ranges (a few hundred meters) the distance measurement uncertainty dominantly affects the overall system performance, while at longer ranges the azimuth uncertainty prevails. In order to perform accurate measurements over longer distances, it is necessary to reduce the azimuth uncertainty. This is achievable either with a high accuracy azimuth measuring device (gyrocompass), which would significantly increase the price of the measuring system, or with a slightly modified use of the GNSS receiver/compass.

To determine the orientation of the measuring system a calibration with a known distant object can be performed. Since the positions of the measuring system and the distant object are known, the true azimuth of the object with respect to the measuring system can be determined. The obtained azimuth 
Table 3. Comparison of measurements and simulation

\begin{tabular}{|c|c|c|c|c|c|c|c|c|c|}
\hline & & \multicolumn{2}{|c|}{$\begin{array}{c}\text { A } \\
\text { Distance: } 130 \mathrm{~m} \\
\text { Azimuth: } 85.5^{\circ} \\
\text { Elevation: } 0.2^{\circ} \\
\end{array}$} & \multicolumn{2}{|c|}{$\begin{array}{c}\text { B } \\
\text { Distance: } 1 \mathrm{~km} \\
\text { Azimuth: } 198.2^{\circ} \\
\text { Elevation: }-0.2^{\circ} \\
\end{array}$} & \multicolumn{2}{|c|}{$\begin{array}{c}\text { C } \\
\text { Distance: } 7.4 \mathrm{~km} \\
\text { Azimuth: } 139.8^{\circ} \\
\text { Elevation: } 1.8^{\circ} \\
\end{array}$} & \multicolumn{2}{|c|}{$\begin{array}{c}\text { D } \\
\text { Distance: } 18.5 \mathrm{~km} \\
\text { Azimuth: } 214.3^{\circ} \\
\text { Elevation: } 2.9^{\circ}\end{array}$} \\
\hline & & Sim. & Meas. & Sim. & Meas. & Sim. & Meas. & Sim. & Meas. \\
\hline \multirow{2}{*}{$\begin{array}{l}\text { Std. } \\
\text { deviation [m] }\end{array}$} & Longitudinal & 1.6 & 0.7 & 1.6 & 0.7 & 1.9 & 0.9 & 4.7 & 2.3 \\
\hline & Lateral & 0.4 & 0.4 & 2.6 & 2.2 & 19.7 & 11.3 & 48.3 & 54.5 \\
\hline RMSE [m] & & 1.7 & 6.3 & 3.1 & 3.2 & 19.8 & 15.8 & 48.5 & 62.9 \\
\hline
\end{tabular}

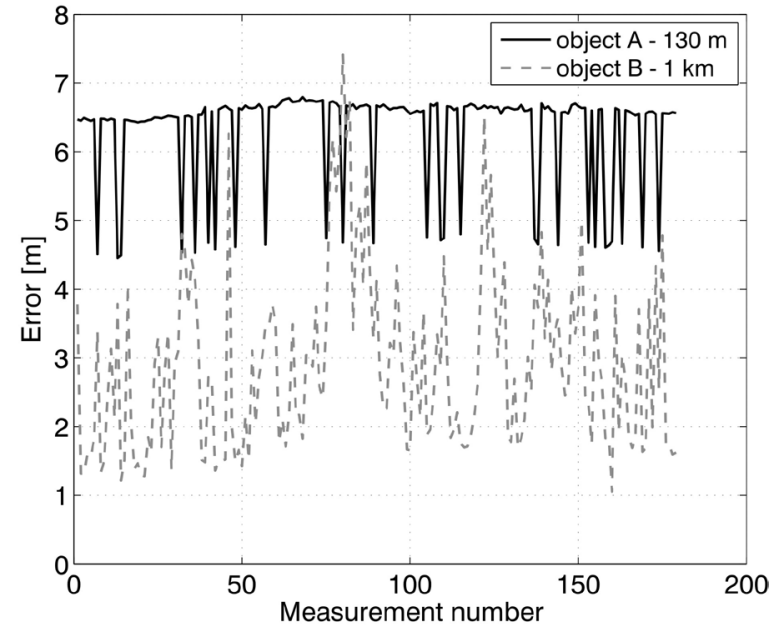

a)

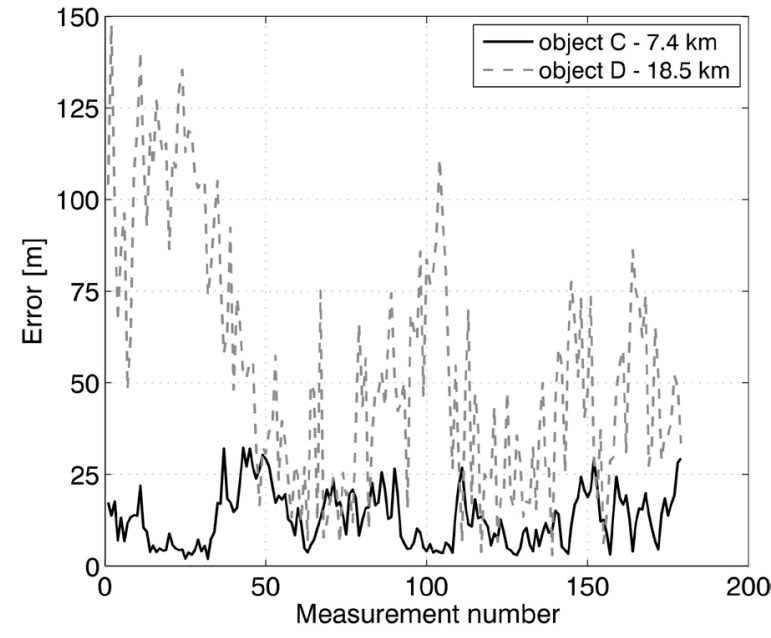

b)

Fig. 8. Measurement errors; a) objects $A$ and $B, b)$ objects $C$ and $D$

value is then used to calibrate the azimuth sensor readings. Such calibration is possible with a geographic information system and has been included in the current version of the control software of the experimental system.

\section{CONCLUSION}

The measurement characteristics of the developed mobile system for remote object geolocation depend on the measurement performances of each employed COTS device. To estimate the overall measurement uncertainty of the measuring system we performed a Monte Carlo simulation with the manufacturer specifications for each COTS device as the simulation input. The comparison of the simulation outputs with the field test results shows that the simulation provides sufficiently accurate estimation of the measurement uncertainty at the distances of up to $7 \mathrm{~km}$. However, at smaller distances of about $100 \mathrm{~m}$ there is a significant bias in the range measurements that limits the system performance. In order to provide better simulation results for shorter ranges, this error needs to be properly characterized and included in the simulation. At longer distances, the azimuth measurement uncertainty of the GNSS compass becomes the main uncertainty source.

Additional set of experiments implied that lower azimuth uncertainties can be achieved using calibration landmarks with known positions. For this purpose it is possible to use existing objects that are represented in a geographic information system or custom calibration landmarks. With the employed GNSS receiver we were able to significantly reduce the azimuth uncertainty with the custom calibration landmark placed only $130 \mathrm{~m}$ away from the measuring system.

The obtained results show that the presented simulation-based methodology for determining the measurement uncertainty of the integrated system is able to provide a satisfactory estimate of the measurement characteristic by relying solely on the manufacturer specification of the employed measuring equipment. 


\section{REFERENCES}

[1] Xu, X., Bhattacharya J.B., Davies, R.K., Aiken, C.L.V. (2001). Digital geologic mapping of the Ferron Sandstone, Muddy Creek, Utah, with GPS and reflectorless laser rangefinders. GPS Solutions, vol. 5, no. 1, p. 15-23, DOI:10.1007/PL00012872.

[2] Madison, R., DeBitetto, P., Rocco Olean, A., Peebles, M. (2008). Target geolocation from a small unmanned aircraft system. IEEE Aerospace Conference, p. 1-19.

[3] Pedlar, D.N., Blake, A.P. (2005). SAR target geolocation performance. IEEE International Radar Conference, $\mathrm{p}$. 212-216, DOI:10.1109/RADAR.2005.1435821.

[4] Lee, J.K., Jekeli, C. (2012). A Dual-IMU/GPS based geolocation system. Journal of Navigation, vol. 65, no. 1, p. 113-123, DOI:10.1017/S0373463311000567.

[5] Hopkinson, C., Chasmer, L., Lim, K., Treitz, P., Creed, I. (2006). Towards a universal lidar canopy height indicator. Canadian Journal of Remote Sensing, vol. 32, no. 2, p. 1-14, DOI:10.5589/m06-006.

[6] Han, K., DeSouza, G.N. (2011). Geolocation of multiple targets from airborne video without terrain data. Journal of Intelligent and Robotic Systems, vol. 62, no. 1, p. 159-183, DOI:10.1007/s10846-010-94427.

[7] Barber, D.B., Redding, J.D., McLain, T.W., Beard, R.W., Taylor, C.N. (2006). Vision-based target geolocation using a fixed-wing miniature air vehicle. Journal of Intelligent \& Robotic Systems, vol. 47, no. 4, p. 361-382, DOI:10.1007/s10846-006-9088-7.

[8] Grejner-Brzezinska, D.A., Toth, C., Sun, H., Wang, X., Rizos, C. (2008). Novel geolocation technology for geophysical sensors for detection and discrimination of unexploded ordnance. IEEE/ION Position, p. 9931007.

[9] Lee, J.K., Jekeli, C. (2011). Rao-Blackwellized unscented particle filter for a handheld unexploded ordnance geolocation system using IMU/GPS. Journal of Navigation, vol. 64, no. 2, p. 327-340, DOI:10.1017/ S0373463310000548.

[10] Kansal, S., Cook, G. (2005). Use of fiducials and unsurveyed landmarks as geolocation tools in vehicular-based landmine search. IEEE Transactions on Geoscience and Remote Sensing, vol. 43, no. 6, p. 1432-1439, DOI:10.1109/TGRS.2005.846152.

[11] Savage, C.O., La Scala, B.F. (2007). Accurate target geolocation using cooperative observers. Information, Decision and Control, p. 248-253, DOI:10.1109/ IDC.2007.374558.
[12] Karimi, H.A., Roongpiboonsopit, D., Kasemsuppakorn, P. (2011). Uncertainty in personal navigation services. Journal of Navigation, vol. 64, no. 2, p. 341-356, DOI:10.1017/S037346331000055X.

[13] Bell, T. (2000). Error analysis of attitude measurement in robotic ground vehicle position determination. Navigation, vol. 47, no. 4, p. 289-296.

[14] El-Rabbany, A. (2002). Introduction to GPS: The Global Positioning System. Artech House Publishers, Boston.

[15] Xu, G. (2007). GPS: Theory, Algorithms and Applications. Springer, Berlin.

[16] Burtch, R. (2006). A comparison of methods used in rectangular to geodetic coordinate transformations. American Congress on Surveying and Mapping, p. 1214.

[17] Kutin, J., Bajsić, I. (2002). An analytical estimation of the Coriolis meter's characteristics based on modal superposition. Flow Measurement and Instrumentation, vol. 12, no. 5, p. 345-351, DOI:10.1016/S09555986(02)00006-7.

[18] Dieck, R.H. (2002). Measurement Uncertainty: Methods and Applications, ISA - The Instrumentation, Systems, and Automation Society, Research Triangle Park.

[19] Gusell, A., Ačko, B., Mudronja, V. (2009). Measurement uncertainty in calibration of measurement surface plates flatness. Strojniški vestnik - Journal of Mechanical Engineering, vol. 55, no. 5, p. 286-292.

[20] Lugarić, L., Majdandžić, L., Škrlec, D. (2010) Countrywide Positioning of Domestic Solar Water Heating Systems using Risk Analysis and Geographical Information System. Strojniški vestnik - Journal of Mechanical Engineering, vol. 56, no. 1, p. 3-17.

[21] Vignat, F., Nguyen, D.S., Brissaud, D. (2012). A Method to Determine the Impact of Geometrical Deviations on Product Performance. Strojniški vestnik - Journal of Mechanical Engineering, vol. 58, no. 9, p. 517-526, DOI:http://dx.doi.org/10.5545/sv-jme.2011.268

[22] JCGM 100:2008 (2008). Evaluation of Measurement Data - Guide to the expression of uncertainty in measurement. Joint Committee for Guides in Metrology, International Organization for Standardization, Geneva.

[23] Castrup, S., Castrup, H.T. (2010). Measurement Uncertainty Analysis Principles and Methods, National Aeronautics and Space Administration, Washington DC.

[24] Geopedia (2011). from http://www.geopedia.si, accessed on 2011-05-07. 